\title{
Effect of Huaier granule on recurrence after curative resection of HCC: a multicentre, randomised clinical trial
}

\author{
Qian Chen, ${ }^{1}$ Chang Shu, ${ }^{2,3,4}$ Arian D Laurence, ${ }^{5}$ Yan Chen, ${ }_{1}^{3,6,7}$ Bao-Gang Peng, ${ }^{8}$ \\ Zuo-Jun Zhen, ${ }^{9}$ Jian-Qiang Cai, ${ }^{10}$ Yi-Tao Ding, ${ }^{11}$ Le-Qun Li, ${ }^{12}$ Yu-Bao Zhang, ${ }^{13}$ \\ Qi-Chang Zheng, ${ }^{14}$ Ge-Liang Xu, ${ }^{15}{ }^{B o}$ Li $^{16}$ Wei-Ping Zhou, ${ }^{17}$ Shou-Wang Cai, ${ }^{18}$ \\ Xi-Yan Wang, ${ }^{19}$ Hao Wen, ${ }^{20}$ Xin-Yu Peng, ${ }^{21}$ Xue-Wen Zhang, ${ }^{22}$ Chao-Liu Dai, ${ }^{23}$ \\ Ping Bie, ${ }^{24}$ Bao-Cai Xing, ${ }^{25}$ Zhi-Ren Fu, ${ }^{26}$ Lian-Xin Liu, ${ }^{27} \mathrm{Yi} \mathrm{Mu}^{28}$ Ling Zhang, ${ }^{29}$ \\ Qi-Shun Zhang, ${ }^{30}$ Bin Jiang, ${ }^{31}$ Hai-Xin Qian, ${ }^{32}$ Yi-Jun Wang, ${ }^{33}$ Jing-Feng Liu, ${ }^{34}$ \\ Xi-Hu Qin, ${ }^{35}$ Qiang Li, ${ }^{36}$ Ping Yin, ${ }^{2}$ Zhi-Wei Zhang, ${ }^{3,6,7}$ Xiao-Ping Chen ${ }^{3,6,7}$
}

\begin{abstract}
Additional material is published online only. To view please visit the journal online (http://dx.doi.org/10.1136/ gutjnl-2018-315983).

For numbered affiliations see end of article.
\end{abstract}

\section{Correspondence to} Dr Ping Yin, Department of Epidemiology and Biostatistics and State Key Laboratory of Environment Health, School of Public Health, Tongji Medical College, HUST, Wuhan, 430030, RR China ; pingyin2000@ 126.com, Dr Zhi-Wei Zhang, Hepatic Surgery Centre at Tongji Hospital of Tongii Medical College of HUST, Jie Fang Avenue 1095, Wuhan 430030, PR China; zwzhang@ tjh.tjmu.edu.cn and Professor Xiao-Ping Chen, Tongji Hospital of Tongji Medical College of HUST, Jie Fang Avenue 1095, Wuhan 430030, PR China; chenxpchenxp@163.com

QC and CS contributed equally.

Received 6 January 2018 Revised 19 April 2018 Accepted 23 April 2018 Published Online First 25 May 2018

\section{ABSTRACT \\ Objective There is little evidence that adjuvant} therapy after radical surgical resection of hepatocellular carcinoma (HCC) improves recurrence-free survival (RFS) or overall survival (OS). We conducted a multicentre, randomised, controlled, phase IV trial evaluating the benefit of an aqueous extract of Trametes robinophila Murr (Huaier granule) to address this unmet need.

Design and results A total of 1044 patients were randomised in 2:1 ratio to receive either Huaier or no further treatment (controls) for a maximum of 96 weeks. The primary endpoint was RFS. Secondary endpoints included OS and tumour extrahepatic recurrence rate (ERR). The Huaier $(n=686)$ and control groups $(n=316)$ had a mean RFS of 75.5 weeks and 68.5 weeks, respectively (HR 0.67; $95 \% \mathrm{Cl} 0.55$ to 0.81). The difference in the RFS rate between Huaier and control groups was $62.39 \%$ and $49.05 \%$ (95\% Cl 6.74 to 19.94; $p=0.0001)$; this led to an OS rate in the Huaier and control groups of $95.19 \%$ and $91.46 \%$, respectively (95\% Cl 0.26 to 7.21; $p=0.0207)$. The tumour ERR between Huaier and control groups was $8.60 \%$ and $13.61 \%(95 \% \mathrm{Cl}-12.59$ to $-2.50 ; p=0.0018)$, respectively.

Conclusions This is the first nationwide multicentre study, involving 39 centres and 1044 patients, to prove the effectiveness of Huaier granule as adjuvant therapy for HCC after curative liver resection. It demonstrated a significant prolongation of RFS and reduced extrahepatic recurrence in Huaier group.

Trial registration NCT01770431; Post-results.

\section{INTRODUCTION}

Hepatocellular carcinoma (HCC) is one of the most common cancers and leading causes of malignancy-related deaths worldwide. ${ }^{1}$ Early-stage HCC can be treated with curative therapies, such as surgery and ablation. However, the overall prognosis is poor with a 5 -year recurrence rate around $70 \%$ either to local recurrence or distant metastasis. ${ }^{23}$ It has been demonstrated that early recurrence of HCC can be

\section{Significance of this study}

What is already known on this subject?

- The primary cause of death after radical surgical resection of hepatocellular carcinoma (HCC) is associated with tumour recurrence.

- Current guidelines do not endorse any particular adjuvant therapy for HCC but recommend larger trials with lower risk of systematic error undertaken.

- Huaier granule is a Chinese State Food and Drug Administration-approved Traditional Chinese Medicine to be used alone or combined with other drugs in treatment of various cancers.

What are the new findings?

- Our study is the first nationwide multicentre study, involving 39 centres and 1044 patients, to prove the effectiveness of Huaier granule as adjuvant therapy for HCC after curative liver resection.

- This phase IV clinical trial showed significant prolongation of recurrence-free survival and reduced extrahepatic recurrence rates in Huaier group.

How might it impact on clinical practice in the foreseeable future?

- The study provides compelling evidence for the effect of Huaier granule as postoperative adjuvant on HCC recurrence and long-term survival.

caused by undetected tumour thrombus invading the inflow or outflow of the hepatic vascular tree, whereas late recurrence results from a de novo second primary tumour. ${ }^{4-6}$ The risk factors associated with recurrence include tumour size, microvascular invasion, multifocality and the absence of tumour capsule, capsule, together with the presence of underlying disease in remnant liver including 
hepatitis virus load, severity of cirrhosis and increased alpha-fetoprotein (AFP), respectively.

The receptor tyrosine kinase inhibitor, sorafenib, was the first drug approved by the Food and Drug Administration to treat unresectable HCC in 2008. However, sorafenib adjuvant therapy following curative resection failed to improve survival in the phase III STORM trial. ${ }^{1}$ Recently, another oral multikinase inhibitor, regorafenib, has been demonstrated to confer a survival benefit for patients with unresectable HCC in the phase III RESORCE trial. Subsequently, it was approved as a secondline treatment for advanced HCC. ${ }^{78}$ Despite these advances, we still lack an effective adjuvant therapy in preventing early recurrence of HCC after potentially curative surgical resection. ${ }^{9}$

Traditional Chinese Medicine (TCM) has a long history for treating cancer in China, and to date, the Guidelines of Diagnosis and Therapy in Oncology using TCM was generated with international standards, to be consistent with modern clinical practice. ${ }^{10}$ Poria robiniophila (Murrill) Ginns (formerly called Trametes robiniophila Murr) or Huaier is a sandy beige mushroom that grows on hard wood trees. It has been widely used in Chinese Medicine for nearly 1600 years. ${ }^{11}$ Huaier granule, the aqueous product of Huaier extract, is an approved TCM by Chinese State Food and Drug Administration (SFDA) to be used alone or combined with other drugs in treatment of leukaemia, osteosarcoma, malignant lymphoma, breast cancer, lung cancer, rectal cancer, liver cancer, gastric cancer, colon cancer and pancreatic adenocarcinoma, respectively. ${ }^{12}$ Recent studies show that the active ingredient in Huaier extract is a proteoglycan, composed of $41.5 \%$ polysaccharides, $12.93 \%$ amino acids and $8.72 \%$ water. $^{13}$ Additional sucrose, dextrin and soluble starch with a 2:2:1 ratio makes up the adjuvants in Huaier granule. Consistent with immunomodulatory chemical structures isolated from other Basidiomycotina mushrooms, Huaier granule modulates innate immunity through stimulating cytokine release and generation of reactive oxygen species and NO. Furthermore, it exerts antitumour responses by inducing cell cycle arrest at the G0/G1 checkpoint and inhibits tumour angiogenesis. ${ }^{14-19}$

Previous clinical studies have suggested that patients with HCC may benefit from postoperative Huaier granule therapy, but this has yet to be proven in a large cohort study with a sufficiently long period of follow-up. ${ }^{20}$ To address this, we conducted a multicentre, randomised, parallel-group controlled, phase IV clinical trial to determine the effect of Huaier granule as postoperative adjuvant to prevent early tumour recurrence.

\section{PATIENTS AND METHODS}

Patients were screened from 39 hospitals in China and 1044 patients were recruited between 7 December 2011 and 31 December 2014. All authors had access to the study data and reviewed and approved the final manuscript.

Site distribution and patient number are described in online supplementary material.

\section{Patient eligibility}

Eligibility criteria included (1) patients (age $\geq 18$ and $\leq 75$ years) with a confirmed first diagnosis of HCC suitable for curative resection treatment according to clinical guidelines ${ }^{21}{ }^{22}$; (2) CT or MRI of chest, abdomen and pelvis performed before surgical resection; (3) the absence of postresection residual tumour confirmed by CT or MRI taken in the first visit during follow-up period; (4) evidence of a histological cancer-free resection margin (defined as the distance between the cancer and the margin that is $1 \mathrm{~cm}$ or more); (5) Barcelona Clinic Liver
Cancer Staging System stage A or B; (6) adequate liver and renal function assessed by laboratory tests done with samples taken within 14 days before randomisation, including alanine aminotransferase less than three times the institutional upper limit of normal $(3 \times \mathrm{ULN})$, aspartate aminotransferase $<3 \times \mathrm{ULN}$, total bilirubin $\leq 2 \times \mathrm{ULN}$, serum creatinine $<1.5 \times \mathrm{ULN}$, haemoglobin $\geq 9 \mathrm{~g} / \mathrm{dL}$, blood platelet count $\geq 60 \times 10^{9} / \mathrm{L}$ and absolute neutrophil count $>1 \times 10^{9} / \mathrm{L}$, respectively.

Exclusion criteria included (1) patients with extrahepatic metastases who underwent hepatic resection even though those metastases were radically resected; (2) tumour metastases or portal invasion or macrovascular invasion confirmed by postoperational histology; (3) Child-Pugh liver function class C; (4) history of abnormal bleeding tendency; (5) known HIV infection; (6) patients with severe acute or chronic disease, including myocardial infarction, stroke, congestive heart failure within 3 months prior to the study, gastrointestinal bleeding within 1 year prior to the study, diabetes and uncontrolled infection prior to the study; (7) history of drug abuse or psychiatric disorders; (8) receiving other investigational or antitumour medications within 4 weeks prior to the study; and (9) women who were pregnant or breast feeding.

\section{Study design}

This was a multicentre, randomised, open-labelled, blank and parallel controlled clinical trial. Huaier granule was obtained from Qidong Gaitianli Pharmaceutical, Jiangsu, China. This product was subject to a phase III clinical trial conducted between March 1993 and June 1994 (Drug Manufacturing Certificate ID: Z20000109). Huaier granule received the first New Drug Certificate (NDC) from SFDA in 2002. Up to 2008, other NDCs have been issued to the approved registration application for claiming various indications, including liver cancer, breast cancer, lung cancer, gastric cancer and rectal-colon cancer. To yield the active ingredient, the dried mushrooms are first grinded to powder, subjected to hot water extraction three times repeatedly applying Sevag reagent to remove unconjugated proteins, followed by further dialysis to remove small molecules and other impurities. Subsequent ethanol precipitation, centrifugation and dehydrolysis result in a 'refined paste', with approximately $1.5 \%-1.8 \%$ proteoglycan yield. ${ }^{132324}$ For raw material preparation and quality control, the chromatographic fingerprint analysis is used to illustrate product's 'phytoequivalence' in manufacturing Huaier extract and ensure the consistency of the active ingredient as required by SFDA. ${ }^{25}{ }^{26}$ Each batch was manufactured to Good Manufacturing Practice standards and followed Quality Assurance Standards required by SFDA (identification code: WS3-215(Z-029)-2001(Z)-2012; YBZ042020032009Z-2012). In the present study, each Huaier granule packet contained $20 \mathrm{~g}$ Huaier extract, which was mixed with $100 \mathrm{~mL}$ of water for oral ingestion. Because it has a distinctive taste that cannot be replicated, we were unable to double blind the trial and control group was designed as a no-treatment (blank control) group. Given absence of medical treatment after curative resection does not contribute much to the overall survival and to encourage recruitment, fewer patients were randomised to the blank control group in the current trial setting resulting in a ratio of $2: 1$. Overall, patients who recovered after curative HCC resection and satisfied the eligibility criteria were subsequently randomised on day 15 to either receive $20 \mathrm{~g}$ Huaier granule orally, three times a day, or to receive no Huaier for 96 weeks. 
Randomisation was centralised through a computer-generated system and performed in a parallel fashion. A data manager, who was not involved in the data analysis or patient enrolment, generated the randomisation schedule. Allocation was concealed by using scratch cards (0001-1044) to receive Huaier or no Huaier in a 2:1 ratio. In detail, each treatment method with an assigned number was printed on a single scratch card, which was stuffed into a blank envelope and further sealed by study coordinators. After baseline assessment and the patient has consented to participate in the study, the study coordinators handed the scratch card in a sealed envelope to study physicians to open. ${ }^{27}$ Study physicians were not blinded to the type of medicine they provided. Patients knew whether they received medicine or not. The randomisation schedule was not available to study recruiters or physicians until baseline assessment was completed. The randomisation remained blinded to the radiologists until the completion of the trial.

First follow-up visit was conducted on week 8 postoperation and individual patients were subsequently asked to visit centres on weeks $16,24,36,48,60,72,84$ and 96, respectively. The follow-up programme included assessment of Eastern Cooperative Oncology Group (ECOG) performance status, Child-Pugh status, serum AFP level, liver function test, renal function test, complete blood counts (CBC), urine test, coagulation test, HBV serology test and HBV viral load test, ultrasonography of the liver, chest radiography and CT scan or MRI examination of liver, respectively. Serious adverse events (SAEs) were recorded whenever noted.

Treatment compliance was evaluated by the gathered information from every follow-up visit, such as whether the patient visited the centre following the schedule and whether he/she documented the daily diary card with $\mathrm{AE}$ or use of concomitant medication on time. Based on the information, each subject was rated 'good compliance' as following the treatment plan and 'low compliance' as either following the treatment but not the schedule or taking other medicine rather than the indicated one. Patients were considered non-adherent if they were rated as 'low compliance' on more than two occasions.

Patients were considered as either withdrawn or dropout cases under the following circumstances: (1) if they were inadvertently enrolled and violating the inclusion/exclusion criteria; (2) if residual HCC was confirmed by CT or MRI within 8 weeks after surgery; (3) if patients were lost to follow-up visits after contacting more than three times; (4) if severe AEs were identified in study population. Patients who had rapid progression of disease and required urgent medical intervention were treated immediately following the local practical guidelines and excluded from the trial.

HBV carriers with cirrhosis and with persistence of viral replication after curative resection were allowed to continue antiviral treatment. This included nucleoside analogues (lamivudine, entecavir and adefovir) and interferon $\alpha$ (IFN $\alpha$ general and Peg-IFN $\alpha$ ) either alone or in combination, following practice guidelines of individual centres.

Risk of recurrence correlated with the following factors was studied, including the number of tumours, tumour size, hepatitis activity, the presence of cirrhosis, poor differentiation of the resected tumour, microsatellite lesion, venous invasion, absence of tumour capsule, positive resection margin and high ChildPugh scores. ${ }^{428} 29$

\section{Endpoints}

The primary endpoint was recurrence-free survival (RFS) and rate of RFS. RFS was defined as the time from randomisation to the first documented disease recurrence by independent radiological assessment or death by any cause, whichever happened first. RFS rate was defined as the incidence of disease recurrence

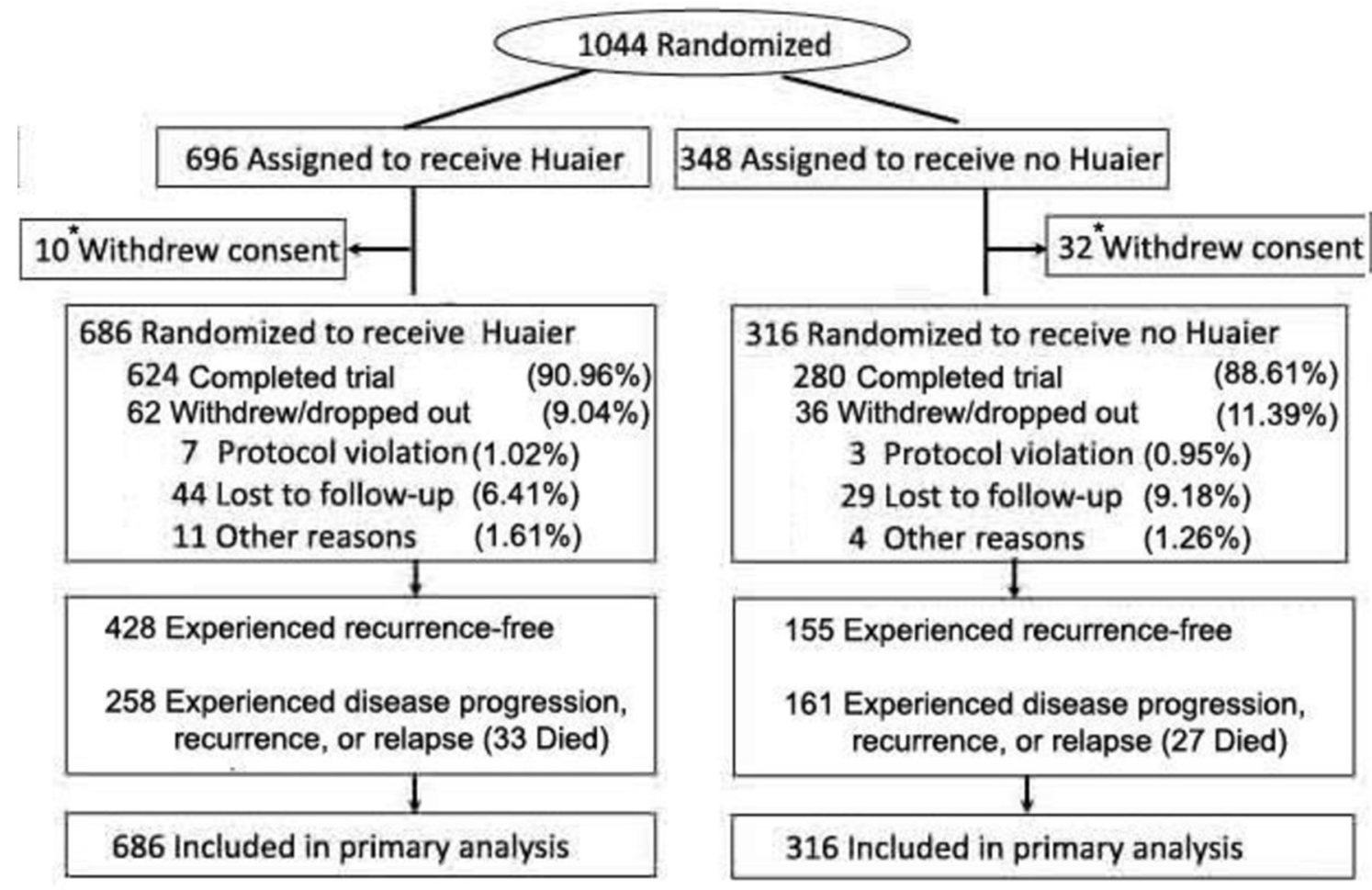

Figure 1 Enrolment and outcomes. * indicates the withdrawal of consent prior to randomisation. There were 10 cases in the Huaier group and 32 cases in the control group who signed consent but did not return after surgery. 
or death by any cause within 96 weeks after randomisation. Secondary endpoints were overall survival (OS) and tumour extrahepatic recurrence rate (ERR). OS was defined as the time from randomisation to death by any cause.

Recurrence was classified as (1) intrahepatic recurrence, defined as a new space-occupying lesion present in the liver on imaging with features of HCC and/or a typical vascular pattern of HCC on dynamic imaging (ie, hypervascularisation in the arterial phase with washout in the portal venous or late venous phase), with or without elevated serum AFP, and (2) extrahepatic recurrence, determined based on Per Response Evaluation Criteria in Solid Tumors. The date of intrahepatic or extrahepatic recurrence was the earliest date at which any one of the above criteria was met. All imaging data (either CT or MRI scans) were collected into a picture/plan film archiving. Two selected radiologists with more than 20 years of experience from Tongji hospital, HUST, and Wuhan Union hospital, HUST, attended the archiving centre and independently reviewed the individual images. Each radiologist received university Standard Operating Procedures training prior to trial and they were blinded to the treatment method. If there was any discrepancy in the interpretation of the hospital CT scans, the two readers would discuss with each other to make a final diagnosis of recurrence after reviewing all clinical materials. The 'recurrence' was reported either as having 'intrahepatic only', 'extrahepatic only' or 'local and systematic' metastasis. After tumour recurrence was confirmed, the patients were informed and managed following practice guidelines of individual centres.

\section{Assessment of safety}

The prescribing information provided in Huaier package gave details of the potential side effects that were principally nausea and emesis although currently there is no causal relationship between this TCM and the listed AE. During the trial, any drug-related $\mathrm{AE}$ was defined as any $\mathrm{AE}$ that occurred when receiving Huaier granule therapy that could be possibly related to the study drug. Serious drug-related AEs were defined as those that resulted in death or required hospitalisation. The occurrence of possible AE was assessed on day 15 after curative liver resection and also during each follow-up visit by interview, physical examination and laboratory tests.

\section{Statistical analyses}

Sample size was determined based on primary objective of comparing RFS between Huaier granule and blank control. Based on expected incidence of recurrence with or without adjuvant treatment, $40 \%$ and $50 \%$, respectively, it required 580 patients in the treatment group and 290 in the control group $(\alpha=0.05, \beta=0.2$, power $=0.8)$. Based on randomised block design, the block number was 9 . We increased the sample size by $20 \%$ to compensate for withdrawal/dropout, leading to a total of 1044 patients: 696 in the treatment group and 348 in the control group. The randomised block design was performed using SAS statistical software (V. 9.3; SAS Institute, USA).

Efficacy endpoints were analysed using 'full analysis set (FAS)' that was used as an approximation of the intention-to-treat population, including all randomly assigned patients. ${ }^{30}$ Schoenfeld residual tests were used to assess proportional hazard assumption and showed no significant correlation between Schoenfeld residual and all three endpoints, demonstrating that the proportional hazard assumption was satisfied (online supplementary table 1). The association between RFS or extrahepatic RFS and those baseline stratification factors were analysed by
Table 1 Baseline demographic and disease characteristics

\begin{tabular}{llll}
\hline & $\begin{array}{l}\text { Huaier group } \\
(\mathbf{n}=686)\end{array}$ & $\begin{array}{l}\text { Control group } \\
(\mathbf{n}=316)\end{array}$ & P values \\
\hline Age (years), $\mathrm{n}(\%)$ & & & \\
$<65$ & $573(83.53)$ & $275(87.03)$ & 0.1537 \\
$\geq 65$ & $113(16.47)$ & $41(12.97)$ &
\end{tabular}

Sex, n (\%)

$\begin{array}{lccc}\text { Male } & 565(82.36) & 255(80.70) & 0.5252 \\ \quad \text { Female } & 121(17.64) & 61(19.30) & \\ \text { Smoking, } n(\%) & & & \\ \quad \text { Never } & 436(63.56) & 208(65.82) & 0.7716 \\ \text { Yes } & 211(30.76) & 92(29.11) & \\ \text { Quitted } & 39(5.69) & 16(5.06) & \end{array}$

Alcohol consumption, $\mathrm{n}(\%)$

\begin{tabular}{|c|c|c|c|}
\hline Yes & $147(21.43)$ & 68 (21.52) & 0.9742 \\
\hline No & 539 (78.57) & $248(78.48)$ & \\
\hline \multicolumn{4}{|c|}{ lo of tumours, $\mathrm{n}(\%)$} \\
\hline Single tumour & 595 (86.73) & 274 (86.71) & 0.7924 \\
\hline
\end{tabular}

Multiple tumours

91 (13.27)

$42(13.29)$

Size of tumour $(\mathrm{cm}), \mathrm{n}(\%)$

$\begin{array}{lcrc}<2 & 55(8.02) & 25(7.91) & 0.1053 \\ \geq 2,<5 & 340(49.56) & 149(47.15) & \\ \geq 5,<10 & 240(34.99) & 100(31.65) & \\ \geq 10 & 51(7.43) & 42(13.29) & \\ \text { Tumour capsule, } \mathrm{n}(\%) & & & \\ \text { Yes } & 537(78.28) & 246(77.85) & 0.8779 \\ \text { No } & 149(21.72) & 70(22.15) & \end{array}$

Child-Pugh classt, $\mathrm{n}(\%)$

$\begin{array}{lrrr}\text { A } & 643(93.73) & 291(92.09) & 0.3366 \\ \text { B } & 43(6.27) & 25(7.91) & \\ \text { BCLC status } \neq, n(\%) & & & \\ \text { A } & 547(79.74) & 239(75.63) & 0.1421 \\ \text { B } & 139(20.26) & 77(24.37) & \end{array}$

Virus infection, $\mathrm{n}(\%)$

$\begin{array}{lrrr}(-) & 129(18.80) & 76(24.05) & 0.2052 \\ \text { HBV } & 544(79.30) & 234(74.05) & \\ \text { HCV } & 8(1.17) & 5(1.58) & \\ \text { Others } & 5(0.73) & 1(0.32) & \\ \text { Edmondson-Steiner grade, } \mathrm{n}(\%) & & & \\ \text { I } & 76(11.08) & 33(10.44) & 0.5081 \\ \text { II } & 412(60.06) & 190(60.13) & \\ \text { III } & 190(27.70) & 84(26.58) & \\ \text { IV } & 8(1.17) & 9(2.85) & \end{array}$

Liver cirrhosis, n (\%)

$\begin{array}{lccc}\text { No } & 213(31.05) & 118(37.34) & 0.0492^{*} \\ \text { Yes } & 473(68.95) & 198(62.66) & \\ \text { Ascites, } \mathrm{n}(\%) & & & \\ \quad \text { No } & 634(92.42) & 290(91.77) & 0.7222 \\ \text { Yes } & 52(7.58) & 26(8.23) & \\ \text { Splenomegaly, n (\%) } & & & \\ \quad \text { No } & 575(83.82) & 263(83.23) & 0.8141 \\ \text { Yes } & 111(16.18) & 53(16.77) & \\ \text { AFP (ng/mL), n (\%) } & & & \\ <400 & 465(67.98) & 201(64.42) & 0.2686 \\ \geq 400 & 219(32.02) & 111(35.58) & \\ \text { Missing (n) } & 2 & 4 & \\ \text { TNM class§, n (\%) } & & & \\ \text { I } & 595(86.74) & 274(86.71) & 0.9876 \\ \text { II } & 49(7.14) & 22(6.96) & \end{array}$




\begin{tabular}{cccc}
\hline Table 1 Continued & & & \\
\hline & $\begin{array}{l}\text { Huaier group } \\
(\mathbf{n}=686)\end{array}$ & $\begin{array}{l}\text { Control group } \\
(\mathbf{n}=316)\end{array}$ & P values \\
\hline III & $42(6.12)$ & $20(6.33)$ & \\
\hline
\end{tabular}

${ }^{*} \mathrm{P}<0.05$.

tChild-Pugh score is calculated by assessing ascites (1 point for none, 2 points for slight and 3 points for moderate), serum bilirubin level (1 point for $<2.0 \mathrm{mg} /$ $\mathrm{dL}, 2$ points for $2.0-3.0 \mathrm{mg} / \mathrm{dL}$ and 3 points for $>3.0 \mathrm{mg} / \mathrm{dL}$ ), serum albumin level (1 point for $>3.5 \mathrm{mg} / \mathrm{dL}$, 2 points for $2.8-3.5 \mathrm{mg} / \mathrm{dL}$ and 3 points for $<2.8 \mathrm{mg} / \mathrm{dL}$ ), prolongation of prothrombin time ( 1 point for $<4 \mathrm{~s}, 2$ points for $4-6 \mathrm{~s}$ and 3 points for $>6 \mathrm{~s}$ ) and encephalopathy (1 point for none, 2 points for grade 1 or 2 , and 3 points for grade 3 or 4). Child-Pugh class A is a total score of 5 or 6 points, and class $B$ is a total score of 7-9 points.

$\neq B C L C$ stage $A$ disease is defined as Child-Pugh classification of $A$ or $B$, an ECOG performance status of 0 and single tumour $\angle 5 \mathrm{~cm}$ or three tumours $<3 \mathrm{~cm}$ each; stage B disease is defined as Child-Pugh classification of A or B, an ECOG performance status of 0 and a multinodular tumour.

§TNM class is based on the American Joint Committee on Cancer, 7th Edition.35 AFP, alpha-fetoprotein; BCLC, Barcelona Clinic Liver Cancer Staging System; ECOG, Eastern Cooperative Oncology Group.

Cox proportional-hazards model to generate HRs and 95\% CIs. Log-rank test was used to compare the Kaplan-Meier survival curve between groups. Rates of recurrence between two groups were conducted by $\chi^{2}$ test. HBV analysis was a post hoc analysis conducted by $\chi^{2}$ test. A two-tailed $\mathrm{p}$ value of 0.05 was considered as statistically significant. HRs and corresponding $95 \%$ CIs were calculated and considered statistically significant when the CI excluded 1.0. The Breslow-Day test for $\chi^{2}$ analysis was used to test the homogeneity across centres on recurrence, death and extrahepatic recurrence.

Safety analyses were performed in all randomly assigned patients who received at least one dose of study drug. Fisher's exact test was used to compare the difference between groups. We used SAS V.9.3 for statistical analysis.

\section{RESULTS}

\section{Demographics}

Between 7 December 2011 and 31 December 2014, 1044 patients from 39 hospitals in China were randomly assigned at a 2:1 ratio: 696 patients in the Huaier group and 348 patients in the blank control group (figure 1). The median follow-up was 73.6 weeks. Ten patients in the Huaier group and 32 in the blank control group missed the first follow-up visit after randomisation and were withdrawn from the trial. Thus, at the time of analysis (25 July 2016), 686 patients in the Huaier group and 316 in the control group were included for FAS analysis.

Baseline demographics and disease characteristics were well balanced between two groups (table 1 and online supplementary table 2). Additionally, the proportion of patients with histologically proven liver cirrhosis in the Huaier group was higher than that in the control group (68.95\% vs $62.66 \%$; $p=0.0492)$. Solitary liver nodule was found in majority of patients $(86.73 \%$ in the Huaier group vs $86.71 \%$ in the control group; $p=0.7924)$. Tumour capsulation associated with preventing HCC invasion and local spread was determined either by imaging or by pathology data. It was detected $78.28 \%$ in the Huaier group versus $77.85 \%$ in the control group $(\mathrm{p}=0.879)$. The median size of tumour was $4 \mathrm{~cm}$ in both treated (range, $0.5-18 \mathrm{~cm}$ ) and control (range, $0.5-20 \mathrm{~cm})$ groups $(\mathrm{p}=0.2076)$. The proportion of tumour size of $10 \mathrm{~cm}$ or above was higher in the control $(13.29 \%)$ than in the Huaier group $(7.43 \%)$, though there was no significant difference in the range of tumour sizes between the groups by Fisher's exact test $(p=0.1053)$. Overall, HBV

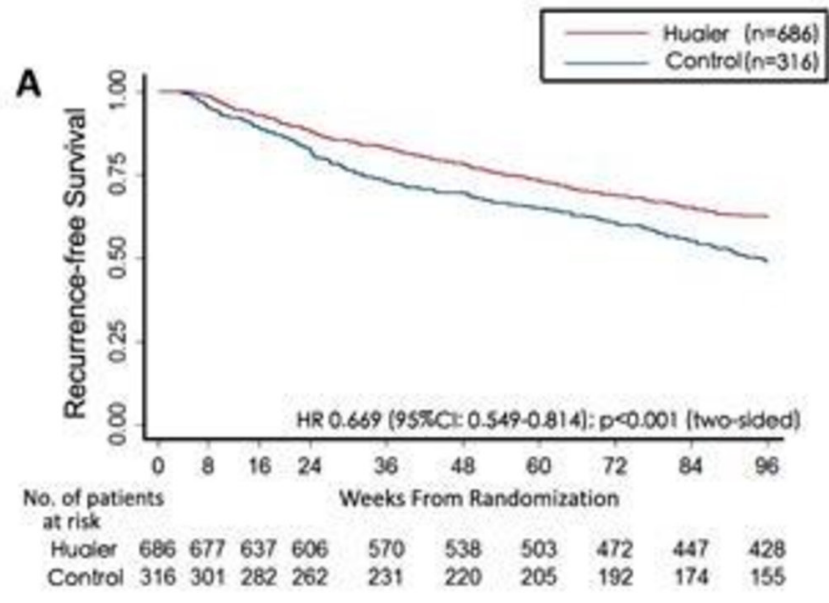

B
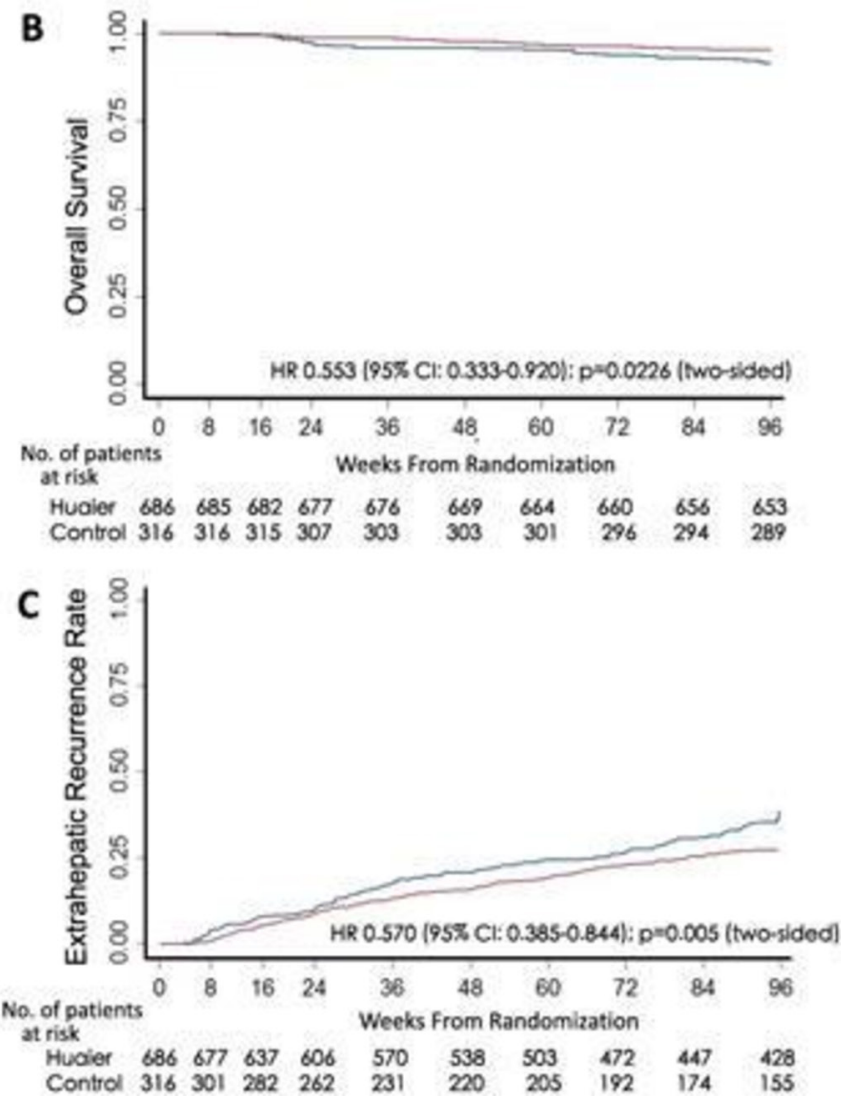

Figure 2 Kaplan-Meier analysis showing (A) recurrence-free survival, (B) overall survival and (C) extrahepatic recurrence rate between Huaier and control groups.

infection was the main cause of HCC and most patients had preserved liver function Child-Pugh status A and TNM class I (table 1).

\section{RFS and time to recurrence}

No patients were reported to have any residual tumour within 8 weeks postoperation. After 96 weeks of follow-up, there were 583 patients who were relapse free (428 in the Huaier group and 155 in the control group) (figure 2). A significantly higher RFS was noted in Huaier group compared with control group (62.39\% vs $49.05 \%, p=0.0001$; figure $2 \mathrm{~A})$. There were 258 patients diagnosed with HCC recurrence in the Huaier group compared with 161 in the control group, resulting in significantly lower recurrence rate in the Huaier group $(37.61 \%$ vs $50.96 \%$; 
Table 2 Comparison of (A) recurrence-free, (B) survival and (C) extrahepatic recurrence between Huaier and control groups

\begin{tabular}{|c|c|c|}
\hline & Huaier $(n=686)$ & Control $(n=316)$ \\
\hline \multicolumn{3}{|l|}{ (A) Recurrence } \\
\hline Recurrence-free, $\mathrm{n}(\%)$ & $428(62.39)$ & $155(49.05)$ \\
\hline Recurrence, $n(\%)$ & $258(37.61)$ & $161(50.56)$ \\
\hline $\mathrm{CMH}-\chi^{2}$ & 14.7315 & \\
\hline$P$ value & 0.0001 & \\
\hline Difference, $95 \% \mathrm{Cl}$ & 13.34 (6.74 to 19.94$)$ & \\
\hline \multicolumn{3}{|l|}{ (B) Survival } \\
\hline Survival, $\mathrm{n}(\%)$ & $653(95.18)$ & $289(91.46)$ \\
\hline Death, $\mathrm{n}(\%)$ & $33(4.81)$ & $27(8.54)$ \\
\hline $\mathrm{CMH}-\chi^{2}$ & 5.3524 & \\
\hline$P$ value & 0.0207 & \\
\hline Difference, $95 \% \mathrm{Cl}$ & 3.73 (0.26 to 7.21$)$ & \\
\hline \multicolumn{3}{|l|}{ (C) Extrahepatic recurrence } \\
\hline No extrahepatic recurrence, $\mathrm{n}(\%)$ & $627(91.40)$ & $273(86.39)$ \\
\hline Extrahepatic recurrence, $n(\%)$ & $59(8.60)$ & $43(13.61)$ \\
\hline $\mathrm{CMH}-\chi^{2}$ & 5.9260 & \\
\hline$P$ value & 0.0149 & \\
\hline Difference, $95 \% \mathrm{Cl}$ & $-7.55(-12.59$ to -2.50$)$ & \\
\hline
\end{tabular}

$\mathrm{CMH}$, Cochran-Mantel-Haenszel test.

$\mathrm{p}=0.0001$, table $2 \mathrm{~A}$ ). Consistently, the mean time to recurrence for the Huaier group was 75.5 weeks, significantly longer than 68.5 weeks for the control group (HR $0.67 ; 95 \% \mathrm{CI} 0.55$ to 0.81; figure $3 \mathrm{~A}$ ). The association between RFS and all baseline stratification factors were analysed by Cox proportional-hazards model. This analysis demonstrated that the most significant benefit from taking Huaier granule was seen in patients with the following characteristics: age $<65$ years, male, the presence of single tumour, tumour size ranging $2-10 \mathrm{~cm}$, tumour capsulation and Child-Pugh class A, respectively (figure 3 ). Huaier granule therapy significantly enhanced RFS for Edmondson-Seiner grade II (HR 0.67; 95\% CI 0.52 to 0.86 ), grade III (HR $0.67 ; 95 \%$ CI 0.46 to 0.97 ) and patients with an AFP $<400 \mathrm{ng} / \mathrm{mL}$ (HR 0.64; $95 \%$ CI 0.50 to 0.83 ), respectively. Furthermore, Huaier granule therapy significantly enhanced RFS in patients with hepatic viral infections, principally $\mathrm{HBV}$ infection (HR $0.67 ; 95 \% \mathrm{CI} 0.53$ to 0.84 ), with cirrhosis (HR $0.76 ; 95 \%$ CI 0.59 to 0.96 ), with no cirrhosis (HR $0.50 ; 95 \% \mathrm{CI} 0.35$ to 0.71 ), with TNM class I (HR $0.65 ; 95 \% \mathrm{CI} 0.52$ to 0.80 ), no ascites (HR $0.66 ; 95 \% \mathrm{CI} 0.54$ to 0.81 ), with splenomegaly (HR $0.72 ; 95 \%$ CI 0.58 to 0.89 ) and without splenomegaly (HR $0.47 ; 95 \% \mathrm{CI} 0.28$ to 0.76 ), respectively (figure 3 ).

Patients who were HBV carriers were allowed to use antiviral treatment during the trial, but not all patients received this therapy. Thus, we were able to compare the effects of antiviral therapy on HCC recurrence rates between the Huaier group and the control group. HBV-positive patients were further stratified into three groups based on whether the antiviral therapy was started before surgery or after surgery or never used. We noticed for those HBV carriers who never received antiviral therapy, Huaier granule treatment resulted in a significant reduction in HCC recurrence $(37.18 \%$ vs $51.82 \%, p=0.0103)$ (table 3$)$.

\section{Overall survival}

Sixty-one patients died: 34 in the Huaier group and 27 in the control group. The 96 -week OS rates were $95.19 \%$ in the Huaier group versus $91.46 \%$ in the control group $(\mathrm{p}=0.0207$, table 2B). Kaplan-Meier analysis demonstrated Huaier group with the superior OS rate to the control group $(p=0.0226)$ (figure 2B). Cox proportional hazards model indicated the HR of 0.553 (95\% Cl 0.333 to 0.92 ), representing a $44.7 \%$ reduction in risk of death.

\section{Extrahepatic recurrence rate}

Extrahepatic recurrence of HCC was associated with a poor survival after surgery. We recorded 59 patients $(8.60 \%)$ with extrahepatic recurrence in the Huaier group and 43 patients $(13.61 \%)$ in the control group $(\mathrm{p}=0.0149)$ (table $2 \mathrm{C})$, including those patients who died from wide systematic spread of tumours and multiorgan failure (four in Huaier vs five in control group). We further observed a significantly lower intrahepatic recurrence rate in Huaier group versus control group by $\mathrm{CMH}-\chi^{2}$ test $(31.20 \%$ vs $39.56 \% ; \mathrm{p}=0.0094)$ (online supplementary table 3). Overall, the ERR was significantly lower in the Huaier group versus control group by Kaplan-Meier analysis (HR $0.570 ; 95 \%$ CI 0.385 to $0.844 ; p=0.005$ ) (figure $2 \mathrm{C}$ ). Huaier adjuvant therapy showed a significant effect on mean extrahepatic recurrence-free survival (ERFS) (89.3 vs 84.3 weeks; HR $0.559 ; 95 \%$ CI 0.403 to 0.774$)$. The association between ERFS and those baseline stratification factors were analysed by Cox proportional-hazards model, indicating the significant adjuvant effects of Huaier group in aspect of age $<65$ years, male, the presence of single tumour, with tumour capsulation, no hepatitis, HBV infection, Child-Pugh class A, with liver cirrhosis, TNM class I, no ascites and with or without splenomegaly, respectively (table 4).

\section{Safety}

Overall, $175(25.5 \%)$ of 686 patients who received Huaier granule and $72(22.8 \%)$ of 348 patients who were in the control group had an AE. All were reported as mild and tolerable. In the Huaier group, the overall incidence of drug-related $\mathrm{AE}$ was $160(23.3 \%)$, including GI, constitutional and respiratory symptoms as well as abnormal liver function and $\mathrm{CBC}$ tests (table 5). The most commonly reported drug-related AE was liver dysfunction that occurred in 48 patients (7.0\%). However, in comparison with the control group, we found no significant difference in the incidence of liver dysfunction in the Huaier group $(\mathrm{p}=0.4405$; table 5$)$.

\section{Compliance and homogeneity}

For treatment compliance measures, we rated $98.5 \%$ of the subjects in the Huaier group and $98.4 \%$ in the control group with 'good compliance', and $1.5 \%$ of the subjects in the Huaier group and $1.6 \%$ in the control group with 'poor compliance', respectively. Fisher's exact test yielded a value of $\mathrm{p}=0.537$, indicating there was no significant difference in treatment compliance between the two groups. To assess the homogeneity of treatment effects across centres, we further used the Breslow-Day test to assess the recurrence, death and extrahepatic recurrence. The $\mathrm{p}$ value was not statistically significant, indicating homogeneous results across centres (online supplementary table 4).

\section{DISCUSSION}

Tumour recurrence represents the primary cause of death after radical surgical resection of HCC. It is associated with tumour size, hepatitis activity, the presence of cirrhosis, poor differentiation of the resected tumour, microsatellite lesion, venous invasion, absence of tumour capsule, positive resection margin and high Child-Pugh scores, respectively. ${ }^{4829}$ The provision of adjuvant therapy for HCC has been limited by both lack of efficacy 


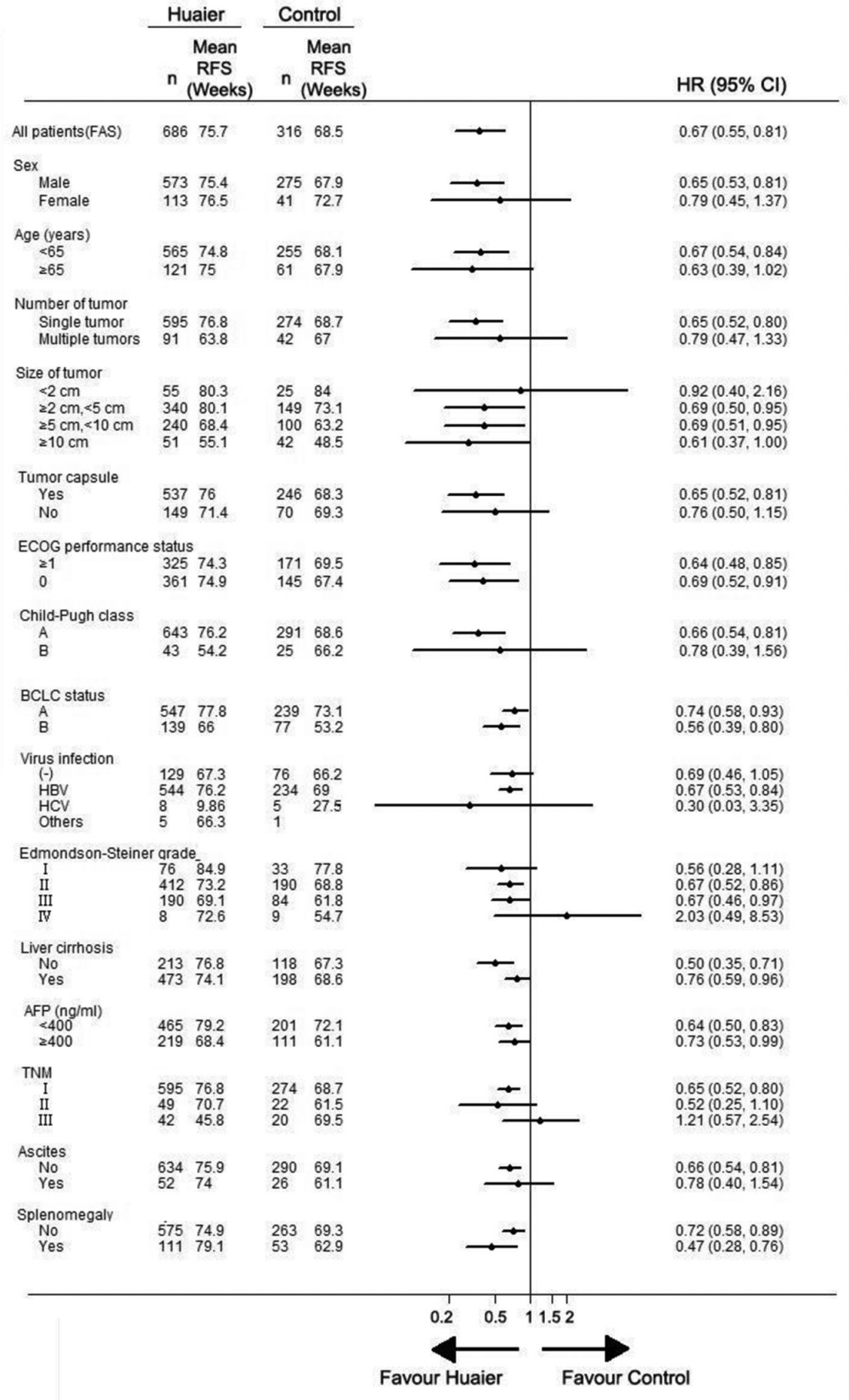

Figure 3 Subgroup analysis of recurrence-free survival (RFS) between Huaier and control groups by Cox regression. BCLC, Barcelona Clinic Liver Cancer Staging System; ECOG, Eastern Cooperative Oncology Group; FAS, full analysis set.

\begin{tabular}{|c|c|c|c|c|c|c|}
\hline \multirow[b]{2}{*}{ Antiviral therapy } & \multicolumn{2}{|l|}{ Huaier $(n=544)$} & \multicolumn{2}{|l|}{ Control (n=234) } & \multirow[b]{2}{*}{$\chi^{2}$} & \multirow[b]{2}{*}{$P$ values } \\
\hline & Recurrence $\mathbf{n}(\%)$ & Recurrence-free $\mathbf{n}(\%)$ & Recurrence $\mathrm{n}(\%)$ & Recurrence-free $\mathbf{n}(\%)$ & & \\
\hline Before surgery only & $67(37.02)$ & $114(62.98)$ & 31 (47.69) & $34(52.31)$ & 2.2742 & 0.1315 \\
\hline After surgery only & $48(37.21)$ & 81 (62.79) & $29(49.15)$ & $30(50.85)$ & 2.3880 & 0.1223 \\
\hline No antiviral therapy & 87 (37.18) & 147 (62.82) & $57(51.82)$ & $53(48.18)$ & 6.5884 & 0.0103 \\
\hline
\end{tabular}


Table 4 Stratified cox regression analysis of extrahepatic recurrence-free survival (ERFS) by groups

\begin{tabular}{|c|c|c|c|c|c|c|}
\hline & \multicolumn{2}{|c|}{ Huaier } & \multicolumn{2}{|c|}{ Control } & \multirow[b]{2}{*}{ HR } & \multirow[b]{2}{*}{$95 \% \mathrm{Cl}$} \\
\hline & $\mathrm{n}$ & $\begin{array}{l}\text { Mean ERFS } \\
\text { (weeks) }\end{array}$ & $\mathbf{n}$ & $\begin{array}{l}\text { Mean ERFS } \\
\text { (weeks) }\end{array}$ & & \\
\hline All patients (FAS) & 686 & 89.3 & 316 & 84.3 & 0.559 & 0.403 to 0.774 \\
\hline \multicolumn{7}{|l|}{ Sex } \\
\hline Male & 573 & 89.4 & 275 & 83.9 & 0.551 & 0.388 to 0.784 \\
\hline Female & 113 & 88.4 & 41 & 86.1 & 0.601 & 0.252 to 1.432 \\
\hline \multicolumn{7}{|l|}{ Age (years) } \\
\hline$<65$ & 565 & 89.3 & 255 & 83.6 & 0.531 & 0.371 to 0.76 \\
\hline$\geq 65$ & 121 & 82.0 & 61 & 87.2 & 0.704 & 0.319 to 1.552 \\
\hline \multicolumn{7}{|l|}{ No of tumours } \\
\hline Single tumour & 595 & 89.6 & 274 & 84.0 & 0.532 & 0.376 to 0.754 \\
\hline Multiple tumours & 91 & 87.3 & 42 & 84.7 & 0.764 & 0.301 to 1.941 \\
\hline \multicolumn{7}{|l|}{ Size of tumour $(\mathrm{cm})$} \\
\hline$<2$ & 55 & 821.8 & 25 & 86.0 & 0.354 & 0.079 to 1.548 \\
\hline$\geq 2,<5$ & 340 & 91.6 & 149 & 89.2 & 0.684 & 0.388 to 1.206 \\
\hline$\geq 5,<10$ & 240 & 77.3 & 100 & 80.3 & 0.554 & 0.335 to 0.918 \\
\hline$\geq 10$ & 51 & 74.6 & 42 & 64.8 & 0.538 & 0.25 to 1.155 \\
\hline \multicolumn{7}{|l|}{ Tumour capsule } \\
\hline Yes & 537 & 88.8 & 246 & 84.3 & 0.606 & 0.42 to 0.874 \\
\hline No & 149 & 83.5 & 70 & 84.3 & 0.406 & 0.196 to 0.84 \\
\hline \multicolumn{7}{|l|}{ Child-Pugh class } \\
\hline A & 643 & 89.9 & 291 & 84.8 & 0.534 & 0.378 to 0.756 \\
\hline B & 43 & 79.6 & 25 & 66.3 & 0.9 & 0.349 to 2.32 \\
\hline \multicolumn{7}{|l|}{ BCLC status } \\
\hline$A$ & 547 & 89.7 & 239 & 88.2 & 0.726 & 0.486 to 1.086 \\
\hline B & 139 & 87.5 & 77 & 71.8 & 0.33 & 0.182 to 0.596 \\
\hline \multicolumn{7}{|l|}{ Virus infection } \\
\hline$(-)$ & 129 & 87.4 & 76 & 85.6 & 0.379 & 0.168 to 0.855 \\
\hline HBV & 544 & 89.6 & 234 & 83.6 & 0.545 & 0.375 to 0.792 \\
\hline $\mathrm{HCV}$ & 8 & - & 5 & - & - & - \\
\hline Others & 5 & - & 1 & - & - & - \\
\hline \multicolumn{7}{|c|}{ Edmondson-Steiner grade } \\
\hline 1 & 76 & 93.6 & 33 & 70.1 & 0.886 & 0.229 to 3.429 \\
\hline II & 412 & 89.0 & 190 & 83.3 & 0.574 & 0.378 to 0.872 \\
\hline III & 190 & 80.8 & 84 & 81.2 & 0.441 & 0.246 to 0.793 \\
\hline IV & 8 & 88.4 & 9 & 19.6 & 2.405 & 0.216 to 26.72 \\
\hline \multicolumn{7}{|l|}{ Liver cirrhosis } \\
\hline No & 213 & 83.1 & 118 & 85.0 & 0.567 & 0.309 to 1.042 \\
\hline Yes & 473 & 88.8 & 198 & 83.4 & 0.546 & 0.371 to 0.804 \\
\hline \multicolumn{7}{|l|}{ AFP (ng/mL) } \\
\hline$<400$ & 465 & 91.5 & 201 & 87.5 & 0.563 & 0.356 to 0.89 \\
\hline$\geq 400$ & 219 & 83.7 & 111 & 76.6 & 0.591 & 0.369 to 0.944 \\
\hline \multicolumn{7}{|l|}{ TNM class } \\
\hline I & 595 & 89.6 & 274 & 84 & 0.532 & 0.376 to 0.754 \\
\hline II & 49 & 94.2 & 22 & 72.6 & 0.376 & 0.053 to 2.684 \\
\hline III & 42 & 56.7 & 20 & 80.5 & 1.087 & 0.370 to 3.189 \\
\hline \multicolumn{7}{|l|}{ Ascites } \\
\hline No & 634 & 89.7 & 290 & 85.5 & 0.548 & 0.386 to 0.778 \\
\hline Yes & 52 & 84.8 & 26 & 39.4 & 0.637 & 0.260 to 1.560 \\
\hline \multicolumn{7}{|l|}{ Splenomegaly } \\
\hline No & 575 & 89.0 & 263 & 85.3 & 0.583 & 0.407 to 0.836 \\
\hline Yes & 111 & 90.5 & 53 & 74.9 & 0.457 & 0.211 to 0.988 \\
\hline
\end{tabular}

The association between ERFS and those baseline stratification factors, including sex, age, number of tumours, size of tumour, with or without tumour capsule, Child-Pugh class, BCLC status, virus infection status, Edmondson-Steiner grade, with or without liver cirrhosis, AFP level, TNM class, ascites and with or without splenomegaly, respectively, was analysed by Cox proportional-hazards model to generate crude HRs and $95 \% \mathrm{Cls}$.

AFP, alpha-fetoprotein; BCLC, Barcelona Clinic Liver Cancer Staging System; FAS, full analysis set. 
Table 5 Adverse events (AEs) and drug-related AEs

\begin{tabular}{|c|c|c|c|}
\hline & \multicolumn{2}{|c|}{ Huaier $(n=686)$} & \multirow{2}{*}{$\begin{array}{l}\begin{array}{l}\text { Control } \\
(n=316)\end{array} \\
\text { AEs }\end{array}$} \\
\hline & AEs & $\begin{array}{l}\text { Drug-related } \\
\text { AEs }\end{array}$ & \\
\hline Overall incidence, $\mathrm{n}(\%)$ & $175(25.5)$ & $160(23.3)$ & $72(22.8)$ \\
\hline \multicolumn{4}{|l|}{ Constitutional symptoms } \\
\hline Fatigue, n (\%) & $2(<1)$ & $2(<1)$ & $2(<1)$ \\
\hline Fever, n (\%) & $9(1.3)$ & $9(1.3)$ & $6(1.9)$ \\
\hline \multicolumn{4}{|l|}{ Laboratory tests } \\
\hline *Abnormal liver functiont, $\mathrm{n}(\%)$ & $51(7.4)$ & $48(7.0)$ & $18(5.7)$ \\
\hline $\begin{array}{r}\text { Mild leucopenia, } n(\%) \\
\left(>3 \times 10^{9} / L \text {, but }<4 \times 10^{9} / L\right)\end{array}$ & $3(<1)$ & $3(<1)$ & 0 \\
\hline${ }^{*}$ Abnormal CBC $\ddagger$ n (\%) & $24(3.5)$ & $21(3.1)$ & $5(1.6)$ \\
\hline \multicolumn{4}{|l|}{ Respiratory symptoms } \\
\hline Cough, $n(\%)$ & $6(<1)$ & $6(<1)$ & 0 \\
\hline${ }^{*}$ Flu-like symptoms, n (\%) & $41(6.0)$ & $41(6.0)$ & $20(7.0)$ \\
\hline \multicolumn{4}{|l|}{ Gl symptoms } \\
\hline Dyspepsia, n (\%) & $3(<1)$ & $2(<1)$ & $1(<1)$ \\
\hline Nausea or emesis, $\mathrm{n}(\%)$ & $13(1.9)$ & $13(1.9)$ & $2(<1)$ \\
\hline Abdominal pain, $\mathrm{n}(\%)$ & $5(<1)$ & $5(<1)$ & $3(<1)$ \\
\hline *Diarrhoea, n (\%) & $35(5.0)$ & $30(4.4)$ & $6(1.9)$ \\
\hline *Any other complaints§ & $49(7.1)$ & $43(6.3)$ & $21(6.6)$ \\
\hline
\end{tabular}

Adverse events, as shown in Medical Dictionary for Regulatory Activities (MedDRA) V.12.0, were classified as being serious or non-serious in accordance with definition adopted by the International Conference on Harmonisation.

* $P$ values were calculated between drug-related AEs in Huaier group and AEs of control group for liver dysfunction, abnormal CBC, flu-like symptoms, diarrhoea and any other complains of $0.4405,0.1712,0.8284,0.0505$ and 0.8204 , respectively. tOne or more than one of the liver function panel results were abnormal. The liver function panel includes alanine aminotransferase, aspartate aminotransferase, total bilirubin, direct bilirubin, albumin, globulin, serum creatinine, etc.

¥One or more than one of complete blood count (CBC) results were abnormal. $\S$ Record all other complaints during the study, including skin rash, nail disorder, back pain, arthralgia, injury, hypertension, orthostatic hypotension, etc.

and toxicity by virtue of the detrimental effect of any cytotoxic or radiation therapy on recovering liver function. It represents an area of great unmet clinical need. Current guidelines do not endorse any particular adjuvant therapy but recommend larger trials with lower risk of systematic error undertaken. ${ }^{128} 31$

In this randomised study, we studied Huaier granule, a widely used TCM, as adjuvant therapy for the patients with HCC after surgical resection. Most patients had early HCC, presented with single tumour, tumour capsulation, Child-Pugh class A, BCLC status A and TNM class I (table 1). The primary endpoint of a significant improvement in RFS rate with Huaier was met $(62.39 \%$ in Huaier vs $49.05 \%$ in control group, $\mathrm{p}=0.0001)$. Correspondingly, RFS of Huaier group was 75.5 weeks, significantly longer than 68.5 weeks of control (HR $0.67 ; 95 \%$ CI 0.55 to 0.81 ). For secondary endpoints, we observed 96 -week survival rate in the Huaier group was higher than in the control group (95.19\% vs $91.46 \% ; p=0.0207)$, and tumour ERR was significantly lower in the Huaier group compared with control $(8.60 \%$ vs $13.61 \%, \mathrm{p}=0.0149$ ) (table 2 ).

TCM is built on a foundation of three millennia of Chinese medical practice, yet few TCMs have shown benefit in randomised control trials ${ }^{20}$ Huaier is a member of Hymenomycetes, Basidiomycotina. This fungus was first recorded by Shi-zhen $\mathrm{Li}$, a famous Chinese practitioner in Ming Dynasty and since then has been widely applied in clinical practice. ${ }^{11}$ Currently, a number of clinical and laboratory studies have shed light on the underlying mechanism of Huaier's anticancer therapy, ${ }^{11-19}$ including its ability to inhibit vascular endothelial growth factor expression to prevent tumour angiogenesis, ${ }^{18}$ its ability to induce cell cycle arrest at the G0/G1 checkpoint, its role in activating caspase cascades and subsequent disruption of tumour cell mitochondrial function. ${ }^{11} 162432$ The active ingredient of Huaier extract, namely Huaier granule, appears as a light-yellow powder through hot-water extraction, ethanol precipitation, deproteinisation and lyophilisation procedures. ${ }^{13}{ }^{24}$ The crude extract is a water-soluble polysaccharide composed of $96.5 \%$ carbohydrate by six sugars, L-fucose, L-arabinose, D-xylose, D-galactose, D-mannose and D-glucose. ${ }^{13} 19$ Botanical polysaccharides are known to enhance host defensive system through binding to macrophage cell-surface receptors, including CD14, complement receptor 3, Toll-like receptors, scavenger receptor and dectin-1, and hence activating innate immunity. ${ }^{11}{ }^{15}$ Strikingly, a common evolutionarily conserved polysaccharide structural backbone is shared between botanical polysaccharides and microbes, suggesting that the two may share a similar signalling pathway for promoting the host inborn defence system and enhancing immunomodulation. ${ }^{15}$

The bulk of the patients studied had chronic hepatitis virus infection. Overall, $\mathrm{HBV}$ infection was an independent risk factor of HCC recurrence. In comparison of $544 \mathrm{HBV}$-infected patients in the Huaier group and $234 \mathrm{HBV}$-infected patients in the control group by Cox regression analysis, Huaier group showed better adjuvant effects on mean ERFS (89.6 vs 83.6 weeks; HR 0.545, $95 \%$ CI 0.375 to 0.792 ) (table 4), as well as mean RFS (76.2 vs 69 weeks; HR $0.67,95 \% \mathrm{CI} 0.53$ to 0.84 ) regardless of the status of cirrhosis (figure 3 ). We further noted that among those who did not receive perioperative antiviral therapy, Huaier treatment showed significant adjuvant effect on tumour recurrence rate $(37.18 \%$ vs $51.82 \%, p=0.0103)$ (table 3$)$. Despite this, we did not see any obvious effect of Huaier on hepatitis B viral load (data not shown). One limitation of the current trial is that for those patients who were HBV carriers, they were allowed to use antiviral treatment during the trial, but not all patients received this therapy. Furthermore, the diversity of antiviral agents in the current trial could not be controlled due to the difference of their healthcare policy and individual financial status. Thus, it is not clear whether Huaier exerts antitumour immunity by mimic antiviral responses. Further research of Huaier plus antiviral therapy versus Huaier alone is warranted.

Our trial design indeed has a limitation on including a small portion of BCLC stage B (20.26\% in the Huaier group and $24.37 \%$ in the control group) (table 1), for which transarterial chemoembolisation currently constitutes the standard of care. ${ }^{21} 22$ Notably, recent studies have demonstrated that BCLC B as intermediate stage includes an extremely diverse set of patients and should be subclassified to facilitate treatment decisions. ${ }^{33}$ In fact, Yamakado et al reported the application of curative treatment such as hepatectomy and radiofrequency ablation in this intermediate stage. ${ }^{34} \mathrm{In}$ the current study, the selected patients who had BCLC stage B but performed with curative hepatectomy were based on the surgeon's experience integrating individual patient preference into treatment decisions from each centre. Therefore, stratification for those intermediate patients of BCLC B subclass in future study will be conducted to ensure the clinical benefit derived from this treatment.

Other limitations of this study include the lack of placebo control and furthermore OS data that resulted from less-than-5-year study. Huaier granule has a distinctive taste, which is widely recognised within the general Chinese population and cannot be replicated, making a reliable placebo impossible. We chose RFS rather than $\mathrm{OS}$ as the primary endpoint over a 2-year follow-up period after curative resection to identify early differences in RFS in the current 
trial setting. Given the absence of available medical treatment that could not contribute to the patients' overall survival after relapse, we assume that the presence of relapse over a 2-year period will closely mirror the OS over a 5-year period.

Together, our study is the first nationwide multicentre study, involving 39 centres and 1044 patients, to prove the effectiveness of Huaier granule as adjuvant therapy for HCC after curative liver resection. This phase IV clinical trial identified significant prolongation of RFS and reduced ERRs in the Huaier group.

\section{Author affiliations}

'Division of Gastroenterology, Department of Internal Medicine at Tongji Hospital, Tongji Medical College, Huazhong University of Science and Technology (HUST), Wuhan, China

${ }^{2}$ Department of Epidemiology and Biostatistics and State Key Laboratory of Environment Health, School of Public Health, Tongji Medical College, HUST, Wuhan, China

${ }^{3}$ Hepatic Surgery Centre at Tongji Hospital, Tongji Medical College, HUST, Wuhan, China

${ }^{4}$ Surgery administrator office at Tongji Hospital, Tongji Medical College, Hust, Wuhan, China

${ }^{5}$ Hematology Department, The Newcastle upon Tyne Hospitals NHS Foundation Trust at Freeman Hospital, Newcastle, UK

${ }^{6}$ Key Laboratory of Organ Transplantation, Ministry of Education and Ministry of Public Health, Wuhan, China

${ }^{7}$ Hubei Province for the Clinical Medicine Research Center of Hepatic Surgery, Wuhan, China

${ }^{8}$ Surgery Department, First Affiliated Hospital of Sun Yat-Sen University, Guangzhou, China

${ }^{9}$ Surgery Department, First People's Hospital, Foshan, China

${ }^{10}$ Surgery Department, Cancer Hospital of Chinese Academy of Medical Sciences, Beijing, China

${ }^{11}$ Surgery Department, Nanjing Drum Tower Hospital Affiliated to Nanjing University Medical School, Nanjing, China

${ }^{12}$ Surgery Department, Cancer Hospital Affiliated to Guangxi Medical University, Nanning, China

${ }^{13}$ Surgery Department, Third Affiliated Hospital of Harbin Medical University, Harbin, China

${ }^{14}$ Surgery Department, Wuhan Union Hospital of HUST, Wuhan, China

${ }^{15}$ Surgery Department, Shengli Hospital Affiliated to Anhui Medical University, Hefei, China

${ }^{16}$ Surgery Department, West China Hospital of Sichuan University, Chengdu, China

${ }^{17}$ Surgery Department, Shanghai Eastern Hepatobiliary Surgery Hospital, Shanghai, China

${ }^{18}$ Surgery Department, Chinese PLA General Hospital of Medical School of Chinese PLA, Beijing, China

${ }^{19}$ Surgery Department, Cancer Hospital Affiliated to Xinjiang Medical University, Urumqi, China

${ }^{20}$ Surgery Department, First Affiliated Hospital of Xinjiang Medical University, Urumqi, China

${ }^{21}$ Surgery Department, First Affiliated Hospital of Shihezi University School of Medicine, Shihezi, China

${ }^{22}$ Surgery Department, China and Japan Union Hospital of Jilin Hospital, Changchun, China

${ }^{23}$ Surgery Department, Shengjing Hospital of China Medial University, Shenyang, China

${ }^{24}$ Surgery Department, Southwest University Hospital, Chongqing, China

${ }^{25}$ Surgery Department, Beijing Cancer Hospital, Beijing, China

${ }^{26}$ Surgery Department, Shanghai Changzheng Hospital, Shanghai, China

${ }^{27}$ Surgery Department, First Affiliated Hospital of Harbin Medical University, Harbin, China

${ }^{28}$ Surgery Department, Beijing Ditan Hospital Affiliated to Capital Medical University, Beijing, China

${ }^{29}$ Surgery Department, Henan Cancer Hospital Affiliated to Zhengzhou University, Zhengzhou, China

${ }^{30}$ Surgery Department, Guangxi Liuzhou Worker's Hospital, Liuzhou, China

${ }^{31}$ Surgery Department, Taihe Hospital Affiliated to Hubei University of Medicine,

Shiyan, China

${ }^{32}$ Surgery Department, First Affiliated Hospital of Suzhou Medical University, Suzhou, China

${ }^{33}$ Surgery Department, Tianjin No.3 Hospital, Tianjin, China

${ }^{34}$ Surgery Branch at the Hospital of Infectious and Contagious Diseases Affiliated to Fujian Medical University, Fuzhou, China

${ }^{35}$ Surgery Department, Third People's Hospital of Changzhou, Changzhou, China
${ }^{36}$ Surgery Department, Tianjin Medical University Cancer Institute and Hospital (TMUCIH), Tianjin, China

Correction notice This article has been corrected since it published Online First. The abstract has been corrected.

Acknowledgements We thank the patients who took part in this trial and their families, as well as study coordinators and staff members at each study site; the statistical staff at the Department of Epidemiology and Biostatistics, School of Public Health, Tongji Medical College, HUST. We thank the donation of Huaie granule from Qidong Gaitianli Pharmaceutical Co. Ltd for the second 24-week and the fourth 24-week of 96 weeks' study period. We thank all the other participating investigators for providing study materials and patients: PB (Southwest University Hospital): L-XL (the First Affiliated Hospital of Harbin Medical University); Y-BZ (the Third Affiliated Hospital of Harbin Medical University); G-LX (Shengli Hospital Affiliated to Anhui Medical University); BJ (Taihe Hospital Affiliated to Hubei University of Medicine); Q-CZ (Wuhan Union Hospital of HUST); X-YW (Cancer Hospital Affiliated to Xinjiang Medical University); X-YP (the First Affiliated Hospital of Shihezi University School of Medicine); J-QC (Cancer Hospital of Chinese Academy of Medical Sciences): L-QL (Cancer Hospital Affiliated to Guangxi Medical University); H-XQ (the First Affiliated Hospital of Suzhou Medical University); Q-SZ (Guangxi Liuzhou Worker's Hospital); B-GP (the First Affiliated Hospital of Sun Yat-Sen University); Y-JZ (Chinese Arsenal and Police (Wujing) General Hospital); HW (the First Affiliated Hospital of Xinjiang Medical University); Z-JZ (Foshan First People's Hospital); C-YW (Shenzhen Second People's Hospital); Y-JW (Tianjin No.3 Hospital); YM (Beijing Ditan Hospital Affiliated to Capital Medical University); TY (Hubei Cancer Hospital); X-WZ (China and Japan Union Hospital of Jilin Hospital); C-LD (Shengjing Hospital of China Medial University); S-WC (Chinese PLA Genera Hospital of Medical School of Chinese PLA); BL (West China Hospital of Sichuan University); Y-TD (Nanjing Drum Tower Hospital Affiliated to Nanjing University Medical School); Z-MW (Xiang Ya Hospital Central South University); Z-RF (Shanghai Changzheng Hospital); B-CX (Beijing Cancer Hospital); LZ (Henan Cancer Hospital Affiliated to Zhengzhou University); T-BL (the Second Affiliated Hospital of Zhejiang University School of Medicine); J-FL (Hospital of Infectious and Contagious Diseases Affiliated to Fujian Medical University); W-PZ (Shanghai Eastern Hepatobiliary Surgery Hospital); QL (Tianjin Medical University Cancer Institute and Hospital (TMUCIH)); C-YS (the Affiliated Hospital of Guizhou Medical University); M-GY (Fujian Cancer Hospital); E-SL (Linyi Cancer Hospital); X-HQ (Third People's Hospital of Changzhou). We are grateful for Professor Dr Hans G Beger (University of Ulm, Germany) for providing critical review of the manuscript and also for the editorial assistance with earlier versions of the manuscript.

Contributors Z-WZ and X-PC were involved in the development of study protocol, study design, data review and interpretation as joint lead investigators of Huaier clinical trial, and both acted as co-lead authors of the Huaier clinical trial report. CS and Z-WZ were both responsible for the provision of patients and data acquisition. $Q C, A D L$ and X-PC were responsible for the review of statistical tables, interpretation of data and review of the report for medical consistency against the study database. ADL, YC, B-GP, Z-JZ, J-OC, Y-TD, L-QL, Y-BZ, Q-CZ, G-LX, BL, W-PZ, S-WC, X-YW, HW, $X-Y P, X-W Z, C-L D, P B, B-C X, Z-R F, L-X L, Y M, L Z, Q-S Z, B J, H-X Q, Y-J W$, J-FL, X-HQ and $\mathrm{QL}$ were the study physician advisers and contributed to data interpretation. CS and PY were the study statisticians and contributed to data analysis. QC wrote the manuscript. All authors provided critical review of the manuscript and approved the final version for publication.

Funding The study was funded by the Major and Special Program of Hepatic Surgery Clinical Study Center of Hubei Province, China (2014BKB089 and 2013BCB026), the National Natural Science Foundation of China (81471612, 81372495 , and 81573262), the Fundamental Research Funds for the Central Universities, HUST (No. 2016YXZD042) and the Twelfth Five-Year Plan for Major and Special Program of the National Science and Technology (2012-2015).

Disclaimer The funders had no role in the study design, data gathering, analysis, interpretation or writing of the report. The corresponding author had full access to all of the study data, and all authors had access upon request. The corresponding authors had final responsibility to submit the manuscript for publication.

Competing interests None declared.

Patient consent Obtained.

Ethics approval The study protocol was approved by Ethics Committee of Tongii Hospital, Tongji Medical College, HUST and all other participating centres. The trial conformed to the ethical guidelines of the Declaration of Helsinki and Good Clinical Practice (GCP) as well as obeyed local laws.

Provenance and peer review Not commissioned; externally peer reviewed.

(c) Article author(s) (or their employer(s) unless otherwise stated in the text of the article) 2018. All rights reserved. No commercial use is permitted unless otherwise expressly granted. 


\section{REFERENCES}

1 Bruix J, Takayama T, Mazzaferro V, et al. Adjuvant sorafenib for hepatocellular carcinoma after resection or ablation (STORM): a phase 3, randomised, double-blind, placebo-controlled trial. Lancet Oncol 2015;16:1344-54.

2 Forner A, Llovet JM, Bruix J. Hepatocellular carcinoma. Lancet 2012;379:1245-55.

3 Lu LC, Cheng AL, Poon RT. Recent advances in the prevention of hepatocellular carcinoma recurrence. Semin Liver Dis 2014;34:427-34.

4 Poon RT, Fan ST, Ng IO, et al. Different risk factors and prognosis for early and late intrahepatic recurrence after resection of hepatocellular carcinoma. Cancer 2000;89:500-7.

5 Wu JC, Huang YH, Chau GY, et al. Risk factors for early and late recurrence in hepatitis B-related hepatocellular carcinoma. J Hepatol 2009;51:890-7.

6 Imamura H, Matsuyama Y, Tanaka E, et al. Risk factors contributing to early and late phase intrahepatic recurrence of hepatocellular carcinoma after hepatectomy. $J$ Hepatol 2003;38:200-7.

7 Ueshima K, Nishida N, Kudo M. Sorafenib-regorafenib sequential therapy in advanced hepatocellular carcinoma: a single-institute experience. Dig Dis 2017;35:611-7.

8 Bruix J, Qin S, Merle P, et al. Regorafenib for patients with hepatocellular carcinoma who progressed on sorafenib treatment (RESORCE): a randomised, double-blind, placebo-controlled, phase 3 trial. Lancet 2017;389:56-66.

9 Gerbes A, Zoulim F, Tilg H, et al. Gut roundtable meeting paper: selected recent advances in hepatocellular carcinoma. Gut 2018;67:380-8.

10 Liu J, Wang S, Zhang Y, et al. Traditional Chinese medicine and cancer: history, present situation, and development. Thorac Cancer 2015;6:561-9.

11 Zou $\mathrm{Y}$, Xiong $\mathrm{H}$, Xiong $\mathrm{H}$, et al. A polysaccharide from mushroom Huaier retards human hepatocellular carcinoma growth, angiogenesis, and metastasis in nude mice. Tumour Biol 2015;36:2929-36.

12 Song $X$, Li Y, Zhang H, et al. The anticancer effect of Huaier (Review). Oncol Rep 2015;34:12-21.

13 Sun Y, Sun T, Wang F, et al. A polysaccharide from the fungi of Huaier exhibits antitumor potential and immunomodulatory effects. Carbohydr Polym 2013;92:577-82.

14 Wasser SP. Medicinal mushrooms as a source of antitumor and immunomodulating polysaccharides. App/ Microbiol Biotechnol 2002;60:258-74.

15 Schepetkin IA, Quinn MT. Botanical polysaccharides: macrophage immunomodulation and therapeutic potential. Int Immunopharmacol 2006;6:317-33.

16 Bao H, Liu P, Jiang K, et al. Huaier polysaccharide induces apoptosis in hepatocellular carcinoma cells through p38 MAPK. Oncol Lett 2016;12:1058-66.

$17 \mathrm{Hu}$ Z, Yang A, Su G, et al. Huaier restrains proliferative and invasive potential of human hepatoma SKHEP-1 cells partially through decreased Lamin B1 and elevated NOV. Sci Rep 2016:6:31298.

18 Wang $X$, Zhang N, Huo Q, et al. Anti-angiogenic and antitumor activities of Huaier aqueous extract. Oncol Rep 2012;28:1167-75.
19 Wang Y, Liu Y, Hu Y. Optimization of polysaccharides extraction from Trametes robiniophila and its antioxidant activities. Carbohydr Polym 2014;111:324-32.

20 Lei JY, Yan LN, Zhu JQ, et al. Hepatocellular carcinoma patients may benefit from postoperative Huaier aqueous extract after liver transplantation. Transplant Proc 2015;47:2920-4.

21 Bruix J, Sherman M. American Association for the Study of Liver Diseases. Management of hepatocellular carcinoma: an update. Hepatology 2011;53:1020-2.

22 European Association for Study of Liver European Organisation for Research and Treatment of Cancer. EASL-EORTC clinical practice guidelines: management of hepatocellular carcinoma. Eur J Cancer 2012;48:599-641.

23 Chen Y, Yao F, Ming K, et al. Polysaccharides from traditional Chinese medicines: extraction, purification, modification, and biological activity. Molecules 2016;21:1705.

24 Zhang N, Kong X, Yan S, et al. Huaier aqueous extract inhibits proliferation of breast cancer cells by inducing apoptosis. Cancer Sci 2010;101:2375-83.

25 Liang Y, Xie P, Chau F. Chromatographic fingerprinting and related chemometric techniques for quality control of traditional Chinese medicines. J Sep Sci 2010;33:410-21.

26 Jiang Y, David B, Tu P, et al. Recent analytical approaches in quality control of traditional Chinese medicines-a review. Anal Chim Acta 2010;657:9-18.

27 Beksinska ME, Joanis C, Smit JA, et al. Using scratch card technology for random allocation concealment in a clinical trial with a crossover design. Clin Trials 2013;10:125-30

28 Bruix J, Sherman M. Practice Guidelines Committee, American Association for the Study of Liver Diseases. Management of hepatocellular carcinoma. Hepatology 2005;42:1208-36.

29 Shah SA, Cleary SP, Wei AC, et al. Recurrence after liver resection for hepatocellular carcinoma: risk factors, treatment, and outcomes. Surgery 2007;141:330-9.

30 Gupta SK. Intention-to-treat concept: a review. Perspect Clin Res 2011;2:109-12.

31 Omata M, Lesmana LA, Tateishi R, et al. Asian Pacific Association for the Study of the Liver consensus recommendations on hepatocellular carcinoma. Hepatol Int 2010;4:439-74.

32 Xu X, Wei Q, Wang K, et al. Anticancer effects of Huaier are associated with downregulation of P53. Asian Pac J Cancer Prev 2011;12:2251-4.

33 Bolondi L, Burroughs A, Dufour JF, et al. Heterogeneity of patients with intermediate (BCLC B) hepatocellular carcinoma: proposal for a subclassification to facilitate treatment decisions. Semin Liver Dis 2012;32:348-59.

34 Yamakado K, Miyayama S, Hirota S, et al. Prognosis of patients with intermediatestage hepatocellular carcinomas based on the Child-Pugh score: subclassifying the intermediate stage (Barcelona Clinic Liver Cancer stage B). Jpn J Radiol 2014;32:644-9.

35 Benson AB, Abrams TA, Ben-Josef $E$, et al. NCCN clinical practice guidelines in oncology: hepatobiliary cancers. J Nat/ Compr Canc Netw 2009;7:350-91. 